\title{
Presymptomatic transmission of COVID-19 in a cluster of cases occurred in confined space: a case report
}

\section{Xinyi Liu}

Hunan Normal University

Jinru Wu

Loudi Center for Disease Control and Prevention

Min Liu

Loudi Center for Disease Control and Prevention

\section{Yingying Dai}

Lianyuan Center for Disease Control and Prevention

\section{Dan Zhou}

Loudi Center for Disease Control and Prevention

\section{Wenqian Li}

Hunan Normal University

\section{Pan LV}

Hunan Normal University

\section{Ning Zhou}

Department of Infectious Diseases, the Second Xiangya Hospital, Central South University

Pa Wu ( $\nabla$ pawu@hunnu.edu.cn )

Hunan Normal University

\section{Case Report}

Keywords: COVID-19, infectivity, confined space, coronavirus, case report

Posted Date: March 19th, 2020

DOI: https://doi.org/10.21203/rs.3.rs-18053/v1

License: (c) (1) This work is licensed under a Creative Commons Attribution 4.0 International License. Read Full License 


\section{Abstract}

Background: COVID-19 is spreading rapidly intercity and international despite rigid public health prevention and control measures been taken.

Case presentation: In a cluster of infection, six out of seven participants engaged in persistent singing and talking in confined space were later diagnosed COVID-19 patients. One of the cases was asymptomatic with no SARS-CoV-2 nucleic acid detected, but positive for anti-SARS-CoV-2 IgG. None of the household contacts was infected during the following month.

Conclusions: This epidemiological evidence revealed that asymptomatic patients transmitted the coronavirus in confined space with relatively high efficiency, suggesting that it is important to provide better ventilation in public buildings to prevent COVID-19 transmission.

\section{Background}

In December 2019, unexplained pneumonia cases were first identified in Wuhan, Hubei Province. These patients were later found to be infected with coronavirus disease-2019 (COVID-19), which quickly spread around in Hubei Province via person-to-person transmission (1). However, there is limited knowledge regarding the rapid transmission of COVID-19, presumed to be spread through respiratory droplets from coughs or sneezes $(2,3)$. It is unclear whether the epidemically quicker spreading of COVID-19 infection in the current outbreak is due to more efficient person-to-person transmission.

Despite public health prevention and control measures been adopted, the COVID-19 infected patients increased exponentially in Wuhan after emerging, with 425 people infected and several health-care workers affected within a month (4). In January, many people who have travel history to Wuhan or direct contact with patients from Wuhan were diagnosed to be COVID-19 positive cases $(1,5,6)$. Cluster of infections or outbreaks such as cluster in Diamond Princess Cruise, church- and hospital-associated clusters in South Korea took place in buildings with high population density but limited ventilation. To improve efficiency in public health prevention and control, investigating the epidemiologic characteristics and transmission dynamics of the sudden outbreak of COVID-19 is needed.

\section{Case Presentation}

We present a cluster of infection with six patients confirmed COVID-19 positive by Loudi Center for Disease Control and Prevention (CDC). The first case (case 1), living in Wuhan for the most of the time, attended activities with people crowded together for a consecutive of three days starting from Jan 10 , 2020. Case 1 drove to Loudi, on Jan 14,2020, together with case 6 and lived with the household of her mother-in-law for the following two days. Case 1 played cards with four distant relatives (cases 3-5 and relative 1) in a confined room in the evening of Jan 14,2020. People who have been in contact with case 1 in a confined space are summarized in Table 1 with infected contact numbered after case and uninfected contact numbered after relative. Case 1 attended a party with a total of 224 people on Jan 15, 
2020, and had lunch with eight friends and relatives. Afterwards, case 1 went to Karaoke with five distant relatives and friends (case2-4, 6 and relative 1) in a confined room of $15 \mathrm{~m}^{2}$ to sing songs for over two hours and gathered for supper. Notably, except for case 6, who drove case 1 to her mother's house, the people gather in the entertainment place did not see each other afterwards.

\section{Table 1: Summary of all participants stayed in confined space with asymptomatic COVID-19 patient}

\begin{tabular}{|lll|}
\hline & Sex & Age \\
\hline Case 1 & Female & 35 \\
\hline Case 2 & Male & 55 \\
\hline Case 3 & Female & 39 \\
\hline Case 4 & Female & 32 \\
\hline Case 5 & Female & 56 \\
\hline Case 6 & Male & 36 \\
\hline Relative 1 & Female & 33 \\
\hline
\end{tabular}

Case 1 developed cough on Jan 17, 2020, however, she felt better after taking some traditional medicine prescribed by local village hospital. Case 1 lived with the household of her mother for the following days. Case 1 attended another party with 232 guests, however, none in the party was infected according to follow-up survey for over a month. On Jan 23, 2020, case 1 got severe with dry cough and went to hospital. Symptom onset of the cluster case is shown in Figure 1 in chronological order. The examination showed a body temperature of $37.6^{\circ} \mathrm{C}$, with white blood cell count of $2.69 \times 10^{9} / \mathrm{L}$, and lymphocyte absolute value of 0.42 . Her CT images showed multi-focal GGO and mixed exudation and consolidation. Nasopharyngeal swab samples of the case 1 was sent to Loudi CDC for nucleic acid detection of COVID19. Each reported COVID-19 positive result was confirmed by two consistent results using RT-PCR test kits from two different manufacturers (Pfizer and Daan Gene).

After the case 1 was administered to hospital, case 2 developed fever, sore throat, fatigue and chills on Jan 23, 2020 and was confirmed RT-PCR positive for COVID-19 nucleic acid. Case 5 and 4 felt Hunan Province and took the train to Fujian Province on Jan 16 and Jan 18, respectively. The CDC of Fujian Province confirmed that patient 4 and 5 are infected with COVID-19 on Jan 29, 2020 and Jan 31, 2020, respectively. Though with asymptomatic with no severe acute respiratory syndrome coronavirus 2 (SARSCoV-2) nucleic acid detected, case 6 was confirmed positive for anti-SARS-CoV-2 immunoglobulin G ( $\mathrm{IgG}$ ) performed with immunochromatography reagent from Wondfo Biotechnology Co. Ltd, which has been approved by the China National Drug Administration, during a study we initiated in February, 2020 to investigate antibodies of close contacts of COVID-19 patients. 


\section{Discussion And Conclusions}

In this cluster of COVID-19 infection, six patients infected had participated in Karaoke and playing cards in confined space of less than $20 \mathrm{~m}^{2}$ with dense population and poor ventilation. In January, 2020, COVID-19 cases outside Hubei Province is still very rare, so we presumed that the local cases acquired SARS-CoV-2 via participating in the two activities in confined space with index patient. The presumed index patient is unclear, as case 1 and case 6 drove to Loudi from Wuhan together, while case 1 contacted the other cases during her presymptomatic phase, case 6 remained asymptomatic with no SARS-CoV-2 nucleic acid detected, but positive for anti-SARS-CoV-2 IgG.

Collectively, we suggest high transmission chance of COVID-19 in relatively confined space. Thus, we suggest providing better ventilation condition in public buildings and halls, including the railway station, hospital, school classroom and office. Moreover, the COVID-19 should be taken with extreme caution in prevention, as the virus is contagious before symptom onset. Finally, we recommend the entertainment place with high population density and poor ventilation condition be taken as the important place to disinfection in still bustling cities and countries.

\section{Abbreviations}

COVID-19: Coronavirus disease-2019

$C D C$ : Center for Disease Control and Prevention

SARS-CoV-2: Severe acute espiratory syndrome coronavirus 2

IgG: Immunoglobulin G

\section{Declarations}

Ethics approval and consent to participate: The study has been approved by the Hunan Normal University institutional review board.

Consent for publication: Written informed consent was obtained from the patients for publication of this Case report. A copy of the written consent is available for review by the Editor of this journal.

Availability of data and materials: Data sharing is not applicable to this article as no datasets were generated or analysed during the current study.

Competing interests: The authors declare that they have no competing interests.

Funding: This work was supported by the National Natural Science Foundation of China (Grant No. 81902094, 81600497), and the Science and Technology Plan Project of Hunan Province (2019RS1036). 
Those grants serves for publication. The funders of the study had no role in the study design, data collection, data analysis, data interpretation, or writing of the manuscript.

Authors' contributions: All authors have read and approved the final manuscript. PW, JW and XL had full access to all of the data in the study and take responsibility for the integrity of the data and the accuracy of the data analysis. XL, JW, ML, YD, DZ, WL and PL acquired, analyzed and interpreted the patient data. PW was a major contributor in writing the manuscript. NZ has critically suggestions in drafting the manuscript.

Acknowledgments: The authors thank Jianjun Liu, Xinliang Liu, Sixuan Long, Zugui Liu, Guodong Deng, Pan Zhu, RongQiang Liu, Ziyan Zeng for their valuable advice and assistance during this investigation, and the Loudi CDC for the COVID-19nucleic acid tests.

\section{References}

1. Chan JF-W, Yuan S, Kok K-H, To KK-W, Chu H, Yang J, et al. A familial cluster of pneumonia associated with the 2019 novel coronavirus indicating person-to-person transmission: a study of a family cluster. 2020.

2. Huang C, Wang Y, Li X, Ren L, Zhao J, Hu Y, et al. Clinical features of patients infected with 2019 novel coronavirus in Wuhan, China. 2020.

3. Peeri NC, Shrestha N, Rahman MS, Zaki R, Tan Z, Bibi S, et al. The SARS, MERS and novel coronavirus (COVID-19) epidemics, the newest and biggest global health threats: what lessons have we learned? 2020.

4. Li Q, Guan X, Wu P, Wang X, Zhou L, Tong Y, et al. Early transmission dynamics in Wuhan, China, of novel coronavirus-infected pneumonia. 2020.

5. Yu P, Zhu J, Zhang Z, Han Y, Huang LJTJoID. A familial cluster of infection associated with the 2019 novel coronavirus indicating potential person-to-person transmission during the incubation period. 2020.

6. Ralph R, Lew J, Zeng T, Francis M, Xue B, Roux M, et al. 2019-nCoV (Wuhan virus), a novel Coronavirus: human-to-human transmission, travel-related cases, and vaccine readiness. 2020;14(01):3-17.

\section{Figures}




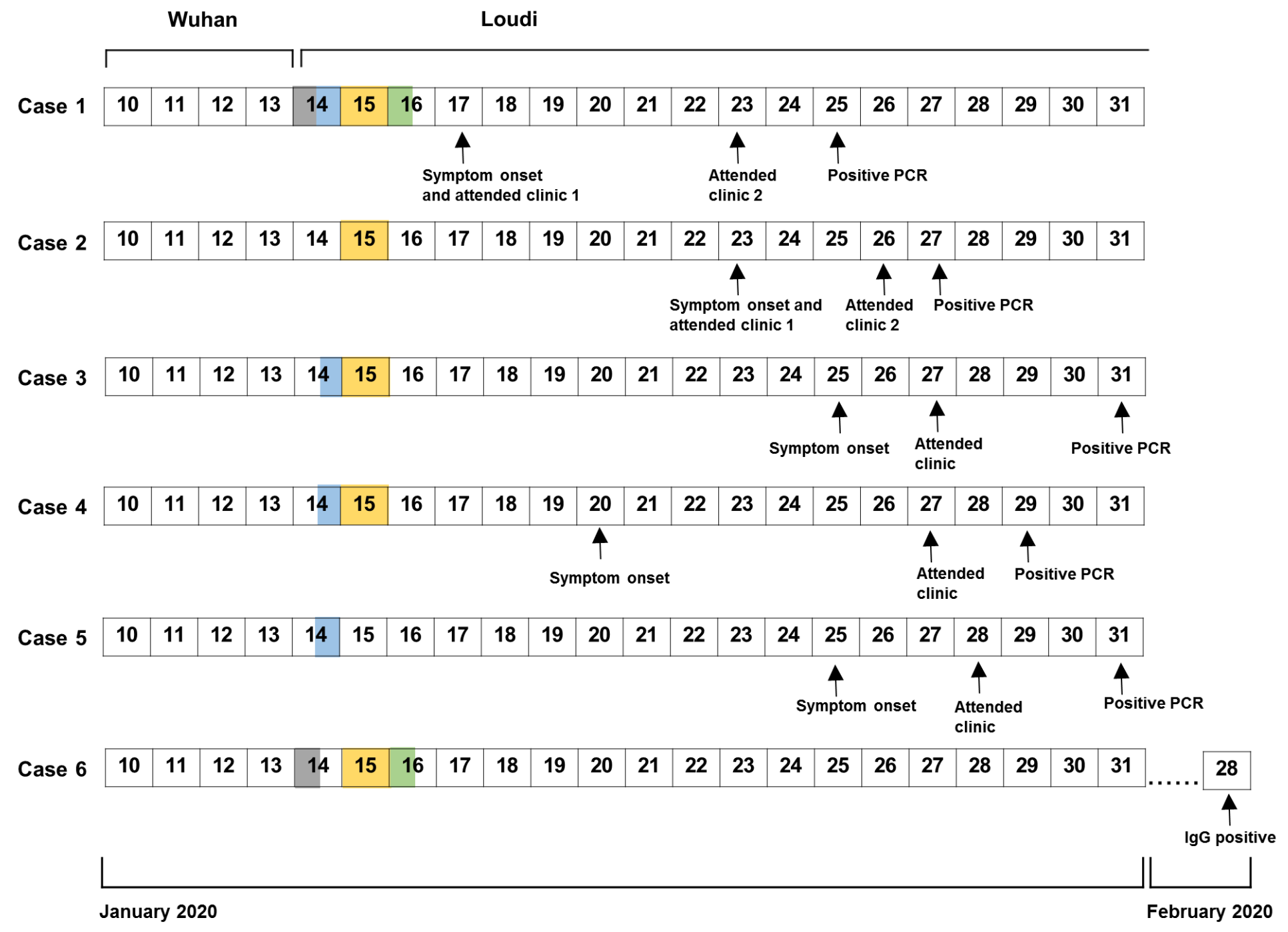

\section{Figure 1}

Timeline of exposure to index patient and dates of symptom onset of the cluster cases. Dates filled in Grey, blue, yellow and green are the dates on which cases 2-6 had close contacts with case 1 . Arrow heads indicates the days on which the clinical events occurred, with the corresponding calendar dates indicated within the box. 